Running head: EMOTION REGULATION STRATEGY USE OVER TIME

Emotion regulation habits related to depression: A longitudinal investigation of stability and change in repetitive negative thinking and positive reappraisal

\author{
Jonas Everaert ${ }^{1 *}$ and Jutta Joormann ${ }^{2}$ \\ ${ }^{1}$ Ghent University, Belgium \\ ${ }^{2}$ Yale University, United States of America
}

* corresponding author: Jonas Everaert, $\mathrm{PhD}$.

Ghent University, Department of Experimental Clinical and Health Psychology, Henri Dunantlaan 2, 9000 Ghent, Belgium. E-mail address: jonas.everaert@gmail.com

Author disclosure: The authors declare that they have no conflict of interest.

Data statement: Anonymized code can be made available for the anonymous reviewers and will be made publicly available via the Open Science Framework upon acceptance of this manuscript. 


\section{Emotion regulation habits related to depression: A longitudinal investigation of stability and change in repetitive negative thinking and positive reappraisal}

Difficulties in regulating negative emotions are theorized to lie at the core of hallmark symptoms of depression (Gross \& Jazaieri, 2014; Joormann, 2010). Two extensively investigated emotion regulation strategies that have been implicated in depression are the habitual use of repetitive negative thinking and positive reappraisal (Aldao, Nolen-Hoeksema, \& Schweizer, 2010; Liu \& Thompson, 2017; Watkins, 2008). Repetitive negative thinking refers to a process of excessive thinking about negative topics that is passive and difficult to control (Ehring et al., 2011; Watkins, 2008). Rumination (focused on past or current distress) and worry (focused on future threats) are two common forms of repetitive negative thinking. Engaging in repetitive negative thinking has been linked to inertia of negative emotions (Waugh et al., 2017), impaired stress recovery (Watkins, 2008), negative cognitive biases (Joormann, Dkane, \& Gotlib, 2006), and less effective problem solving (Nolen-Hoeksema, Wisco, \& Lyubomirsky, 2008). Both subclinical and clinical variants of depression are characterized by higher levels of repetitive negative thinking (Aldao et al., 2010).

Positive reappraisal involves reinterpreting the meaning (i.e., generating alternative meanings) of a negative emotion-eliciting stimulus in a less negative and more positive manner to reduce its emotional impact (Garnefski \& Kraaij, 2007; Ochsner \& Gross, 2008). ${ }^{1}$ Habitual use of positive reappraisal has been associated with increased positive emotions (Brans, Koval, Verduyn, Lim, \& Kuppens, 2013), more positive interpretations of ambiguity (Everaert et al., 2017), better stress recovery (Jamieson, Nock, \& Mendes, 2012), and improved psychological adjustment (Nezlek \& Kuppens, 2008). A lower propensity to use positive reappraisal has been consistently associated with subclinical and clinical forms of depression (Aldao et al., 2010).

\footnotetext{
${ }^{1}$ This study focused on one commonly-studied form of reappraisal (reinterpretation) and did not consider other forms such as distancing (viewing an emotional stimulus from a perspective of a distant observer; McRae, Ciesielski, \& Gross, 2012; Ochsner \& Gross, 2008).
} 
Importantly, longitudinal research has shown that repetitive negative thinking predicts increases in depressive symptoms as well as the maintenance and relapse of clinical depression (Raes, 2012; Spinhoven, van Hemert, \& Penninx, 2018; Topper, Molenaar, Emmelkamp, \& Ehring, 2014). Conversely, habitual positive reappraisal use is related to prospective decreases in depressive symptoms (Brewer, Zahniser, \& Conley, 2016; Garnefski \& Kraaij, 2007; Haga et al., 2012). Together, accumulated evidence indicates that tendencies to use more repetitive negative thinking and less positive reappraisal may confer risk to experiencing depression.

\section{Stability and change of emotion regulation habits}

Though research has made important progress in understanding the role of emotion regulation habits in depression, little is known about one of their theorized core characteristics: the enduring nature. Indeed, theorists have conceptualized the propensity to use repetitive negative thinking or positive reappraisal as individual dispositional characteristics that are stable over time. That is, repetitive negative thinking (Hertel, 2004; Nolen-Hoeksema et al., 2008; Smith \& Alloy, 2009) and positive reappraisal (Gross \& John, 2003; Liu \& Thompson, 2017) are assumed to reflect trait-like tendencies or "habits of thought" that occur frequently and repetitively in response to stress. Indeed, many studies have examined the role of momentary (i.e., state-like) emotion regulation strategy use in distorted emotion dynamics using ecological momentary assessment methods (e.g., Brans et al., 2013; Haines et al., 2016). However, knowledge of the temporal stability of habitual (i.e., trait-like) emotion regulation strategy use seems particularly important in determining whether these emotion regulation processes represent enduring risk for psychological maladaptation.

Some studies examined the temporal stability of habitual repetitive negative thinking and positive reappraisal using two-wave test-retest designs. This research found moderate testretest correlations for repetitive negative thinking (Smith \& Alloy, 2009) and positive reappraisal (Garnefski, Kraaij, \& Spinhoven, 2001), suggesting modest stability in the degree 
to which individuals maintain their relative position regarding their use of these regulatory strategies over time. However, two-wave test-retest designs are limited in their ability to illuminate processes of stability and change because such designs confound true change with measurement error and cannot describe individual trajectories of change (Singer \& Willett, 2003). Minimum three waves of data collection are required to investigate these fundamental aspects of stability and change in emotion regulation habits (Singer \& Willett, 2003).

To date, few studies have employed multi-wave designs to investigate longitudinal trajectories of habitual emotion regulation strategy use. Available studies have exclusively focused on longitudinal change in rumination. In a community sample of adolescents with varying depressive symptom levels, one study measured rumination across four repeated assessments separated by five-week intervals (Hankin, 2008). The results revealed a moderate average test-retest correlation for rumination, suggesting modest stability in the relative rank of individuals on habitual rumination. Moreover, growth modeling showed that the mean level of rumination decreased across the four waves of data collection, indicating that rumination may change over time. While individual differences in rumination emerged at the start of the study, no differences among individuals occurred in the linear change over time. This suggests that adolescents have comparable trajectories of decreasing rumination use regardless of their initial levels. Another study followed community adolescents reporting varying depression levels over the course of three years with yearly assessments of rumination (Mazzer, Boersma, \& Linton, 2019). The results of the structural equation models showed moderately strong relations between two subsequent measurements, providing further evidence for some rank-order stability in habitual rumination. However, different from earlier work (Hankin, 2008), the level of rumination increased linearly over the course of the three-year follow-up. Finally, one recent study in healthy, anxious, and depressed adults examined the stability of rumination across five follow-up assessments over the course of nine years (Struijs et al., 2020). This study did not 
estimate individual change trajectories but computed intraclass correlation coefficients (ICCs) to examine the absolute agreement between scores at two time points (i.e., the extent to which scores at wave 1 equal scores at wave 2, 3, 4 or 5). The results revealed moderate ICCs with a slight decline as the length of the follow-up increased, providing evidence for temporal stability over short and long time intervals.

Taken together, current research suggests modest stability in the relative ranking of individuals on habitual rumination and linear changes in the use rumination over time, while other research indicates that rumination may be stable with decreasing stability over longer time intervals. Accordingly, empirical research on rumination yields mixed support for a stable trait perspective on this emotion regulation process.

\section{Sources of change in trajectories of emotion regulation habits}

Despite initial research on trajectories of emotion regulation habits, the factors that drive change within such longitudinal trajectories are not well understood. Transactional models of stress and vulnerability to depression propose that emotion regulation habits are dynamically related to stressors and depressive symptoms over time (Hankin \& Abramson, 2001; Watkins \& Roberts, 2020). That is, stressors and depressive symptoms may increase engagement in habitual emotion regulation to reduce the experienced distress. When emotion regulation habits are characterized by more repetitive negative thinking or less positive reappraisal, habits may in turn elicit higher levels of stress and increase depressive symptom severity.

Providing initial support for this notion, emerging research on rumination in adults has shown that perceived stress is related to increased rumination over time (Michl, McLaughlin, Shepherd, \& Nolen-Hoeksema, 2013) and that rumination predicts elevations in stress (Flynn, Kecmanovic, \& Alloy, 2010). Furthermore, recent work demonstrated that rumination is related to increases in depressive symptoms which in turn predict increases in rumination (Whisman, du Pont, \& Butterworth, 2020). However, research has yet to formally test whether stress and 
depressive symptoms directly modulate the longitudinal course of repetitive negative thinking and other emotion regulation habits over time.

\section{The present study}

The purpose of this study was twofold: (a) to characterize the prototypical individual trajectories of repetitive negative thinking and positive reappraisal over time, and (b) to examine perceived stress and depression symptoms as sources of temporal change in emotion regulation habits. Consistent with views on emotion regulation habits as stable traits (Liu \& Thompson, 2017; Smith \& Alloy, 2009), we expected that the prototypical longitudinal trajectory of repetitive negative thinking and positive reappraisal would be relatively stable over time. We anticipated individual differences in the initial levels of emotion regulation habits (cf. Hankin, 2008), but limited variability in the rate of change if such habits reflect stable individual traits. Nevertheless, based on prior research observing increasing and decreasing trends (Hankin, 2008; Mazzer et al., 2019) as well as modest test-retest stability (Garnefski et al., 2001; Smith \& Alloy, 2009), we deemed it plausible to observe some change in emotion regulation habits.

Examining within-person sources of variation in longitudinal trajectories, we predicted that both depressive symptoms and perceived stress have time-varying effects on the course of emotion regulations habits (Michl et al., 2013; Whisman et al., 2020). Specifically, we expected that depressive symptoms and perceived stress would predict more repetitive negative thinking and less positive reappraisal at the same wave. Finally, to examine the temporal precedence among these variables, we predicted that emotion regulation habits have bidirectional relations with perceived stress and depressive symptoms (Hankin \& Abramson, 2001). In particular, we anticipated that depressive symptoms and perceived stress predict increases in repetitive negative thinking but decreases in positive reappraisal. We expected that repetitive negative thinking and positive reappraisal use are in turn to be related to increases and decreases in perceived stress, respectively. 


\section{Method}

\section{Participants and sampling strategy}

This study employed a dimensional approach that considered individual differences in repetitive negative thinking and positive reappraisal along full range of depressive symptom severity levels (Insel et al., 2010). Therefore, recruitment of participants for this study was unselected. Participants were recruited through Amazon's Mechanical Turk (MTurk) between October 2016 and July 2017. MTurk provides an online crowdsourcing platform with access to large samples that are suitable for research on psychopathology (Chandler \& Shapiro, 2016; Ophir, Sisso, Asterhan, Tikochinski, \& Reichart, 2018). Participation in the study was restricted to MTurk users who were 18 years $\left(M_{\mathrm{age}}=39.25, S D=12.30\right)$ or older and resided in the United States of America. A total of 320 MTurk users qualified for inclusion in this study. Table 1 provides demographic information. Following recommendations for research using crowdsourced samples (Chandler \& Shapiro, 2016), several steps were taken to ensure high data quality and power. Data quality requirements are detailed in supplement 1.

\section{Procedure}

This longitudinal study consisted of twenty waves of data collection separated by oneweek time intervals, resulting in a total duration of five months. The duration of the follow-up was comparable to prior work (Hankin, 2008), while the one-week time intervals increased the temporal resolution to detect potential fluctuations in habitual emotion regulation strategy use that cannot be captured when using longer time-intervals. At wave 1, participants completed a survey which began with demographic questions (age, gender, race, education level) followed by well-established questionnaires (detailed below). At all subsequent waves, participants completed the same test battery of questionnaires. The questionnaires were presented in randomized order. The study procedures were approved in accordance with the Institutional Review Board at Yale University. All participants gave informed consent after the study 
protocol was explained. Participants were remunerated per survey and up to a total of 15.20 USD for completing all waves of data collection.

\section{Questionnaires $^{2}$}

Participants were instructed to complete the questionnaires in reference to the past week. This was to standardize the temporal orientation across all questionnaires and waves of data collection. Supplement 2 details the instructions. The reliability of the questionnaires to capture within-person changes was calculated following reliability measures described by Bolger and Laurenceau (2013). The coefficients $\left(R_{c}\right.$ 's) are reported below.

\section{Dispositional emotion regulation strategy use}

Habitual repetitive negative thinking. The repetitive negative thinking subscale of the Repetitive Thinking Questionnaire (McEvoy, Mahoney, \& Moulds, 2010) is a transdiagnostic measure of the tendency for perseverative negative thinking. All 27 items of the subscale are scored on a five-point scale from 1 ('not true at all') to 5 ('very true') in reference to distressing situations during the past week. Psychometric research evaluating the repetitive negative thinking subscale in nonclinical and clinical samples has demonstrated that the subscale has a good to excellent high internal consistency as well as validity (Mahoney, McEvoy, \& Moulds, 2012; McEvoy et al., 2010). The total score on the subscale was rescaled so that scores ranged from 0 to 108 to aid interpretation of the growth curve plots. In this study, the subscale had acceptable reliability $\left(R_{c}=.70\right)$ in measuring within-person changes over time as well as excellent internal consistency (Cronbach's $\alpha$ varied between .97 and .98 across waves).

Habitual positive reappraisal. The habitual use of positive reappraisal was measured using the subscale of the Cognitive Emotion Regulation Questionnaire (Garnefski et al., 2001). This 4-item scale specifically measures the use of positive reappraisal in response to negative

\footnotetext{
2 The current study was part of a larger longitudinal study examining emotion regulation in depression and anxiety. In addition to the measures described in this manuscript, we administered the Beck Anxiety Inventory (Beck, Epstein, Brown, \& Steer, 1988). The research questions addressed within this study were a priori.
} 
events during the past week. On each of the 4 items, respondents rate the extent to which they engage in positive reappraisal using a 5-point scale from 1 ('almost never') to 5 ('almost always'). The positive reappraisal subscale has good to excellent internal consistency, acceptable test-retest reliability, and both convergent and divergent validity (Garnefski \& Kraaij, 2007; Garnefski et al., 2001; Ireland, Clough, \& Day, 2017). The total score of the positive reappraisal subscale was rescaled so that scores ranged from 0 to 16 to facilitate interpretation of the growth curve plots. In the present study, the $R_{c}$ coefficient of .84 suggests that the positive reappraisal subscale had good reliability in measuring within-person changes over time. In addition, the internal consistency (Cronbach's $\alpha$ ) varied between .90 and $\alpha=.96$.

\section{Depressive symptom severity}

The Beck Depression Inventory - II (BDI-II; Beck et al., 1996) is the most frequently used self-report instrument to measure the severity of common depressive symptoms. On 21 items, respondents indicate the degree to which they have experienced a certain symptom on a four-point scale from 0 to 3 , yielding a total score ranging from 0 to 63. Participants completed all items in reference to the past week. The reliability and validity of the BDI-II has been extensively supported in both nonclinical and clinical adult samples (Joiner, Walker, Pettit, Perez, \& Cukrowicz, 2005). In this study, the $R_{c}$ coefficient of .81 suggests that the BDI-II had good reliability in measuring within-person changes in depressive symptom severity over time. The internal consistency of the BDI-II varied between Cronbach's $\alpha=.95$ and $\alpha=.96$.

\section{Perceived stress}

The Perceived Stress Scale (Cohen, Kamarck, \& Mermelstein, 1983) is one of the most commonly-used measures of the perception of stress. On each of the 10 items of this questionnaire, respondents indicate the degree to which they appraise situations in their lives as stressful on a five-point scale from 0 ('never') to 4 ('very often'). Participants completed all items in reference to the past week. The total score ranges from 0 to 40 . Research has shown 
that the questionnaire has an adequate internal consistency (Roberti, Harrington, \& Storch, 2006) and convergent validity (Pbert, Doerfler, \& DeCosimo, 1992). In this study, the $R_{c}$ coefficient of .60 indicates that the Perceived Stress Scale has acceptable reliability in measuring within-person changes in perceived stress over time. The internal consistency of the scale varied between Cronbach's $\alpha=.91$ and $\alpha=.94$.

\section{Missing data}

On average, participants completed 17 of the 20 waves of data collection $(S D=5.29)$. Complete data were available for $75 \%$ of the participants. The overall level of missing values was low (15\%) given the intensity and duration of this online longitudinal study. Logistic regression analysis tested whether demographic characteristics (age, gender, race, education), emotion regulation habits (repetitive negative thinking, positive reappraisal), and clinical variables (perceived stress, depressive symptoms) predicted the likelihood of incomplete data $(0=$ incomplete, $1=$ complete $)$. Older age was associated with greater likelihood of complete data, $b=0.04$, Wald's $Z=8.14, p=.004$, Odds Ratio $=1.04$. No other variables were predictive of (in)complete data. Therefore, age was included as a covariate in the analyses to reduce the possibility of bias and permit valid generalizations (Graham, 2009; Singer \& Willett, 2003).

\section{Analytical plan}

Multilevel modeling was used given the hierarchically nested data structure with waves of data collection $(i: 1-20)$ nested within individuals $(j: 1-320)$. This approach is particularly suited to handle unbalanced and missing data as well as within-person observation dependency (Singer \& Willett, 2003). Maximum likelihood method was used to estimate the models. Analyses were conducted with R 3.6.2 (R Core Team, 2018) using the package nlme (Pinheiro, Bates, DebRoy, Sarkar, \& Team, 2018).

To examine the longitudinal trajectories of emotion regulation habits, individual growth curve models were fitted separately for repetitive negative thinking and positive reappraisal. 
First, we estimated an unconditional mean model (Model 1) to examine individual variation in habitual emotion regulation strategy use across individuals without regard to time. The intraclass correlation (ICC) was computed to decompose the total variance into between-person (level-2) and within-person (level-1) variance components. Second, we specified a unconditional linear growth model (Model 2) to investigate systematic change in habitual emotion regulation strategy use over time. The Wave variable was centered on the first measurement occasion (the first wave was coded as 0 ). The intercept and slope were allowed to vary across individuals. A first-order autoregressive covariance structure accounted for higher correlations among repeated assessments that are closer in time and lower correlations with increasing distance between repeated assessments (Singer \& Willett, 2003). Third, to investigate within-person sources of variation in longitudinal trajectories, we tested a conditional linear growth model with perceived stress and depressive symptom severity as time-varying covariates (Model 3). This model examines time-varying effects of perceived stress and depressive symptom severity on trajectories of habitual emotion regulation. Both covariates were within-person centered before added to Model 3. Fourth, in Model 4, the grandmean centered age variable was added as another level-2 predictor to Model 3 to control for its potential relation with individual differences in the initial levels of habitual emotion regulation strategy use (i.e., the intercept) and the rate of change (i.e., the slope) over time. Finally, as in prior work (Hankin, 2008), unconditional mean unconditional linear growth models were also fitted for depressive symptom severity and perceived stress to provide a benchmark against which longitudinal trajectories of emotion regulation habits can be compared.

To examine the temporal precedence between changes in emotion regulation habits and changes in both perceived stress and depressive symptoms, four within-person cross-lagged models were tested. In these models, perceived stress (or depressive symptom severity) at wave $w$ was entered as a predictor of habitual emotion regulation (i.e., repetitive negative thinking or 
positive reappraisal) at the next wave $(w+1)$, controlling for the habitual use of that emotion regulation strategy at wave $w$. The inverse relation was also tested: Habitual emotion regulation at wave $w$ was entered as a predictor of changes in perceived stress (or depressive symptom severity) from wave $w$ to wave $w+1$. Following recommendations to obtain unbiased estimates of the effects of a predictor on the outcome (Hamaker, Kuiper, \& Grasman, 2015), the predictors were decomposed into time-invariant (i.e., an individual's mean score across available waves) and time-varying (i.e., within-person fluctuations from wave to wave around an individual's mean score) components. In light of the current study's focus on within-person dynamics, the results focus on the within-person components even though the between-person components were included in all analyses (as predictors of the random intercept). Note that the analyses controlled for the growth curve of each variable (by adding the wave variable to the models) and included age as a predictor of the random intercept.

\section{Results}

\section{Sample characteristics}

Participants' scores on measures of repetitive negative thinking, positive reappraisal, perceived stress, and depressive symptom severity represented almost the full range of scores at each wave of data collection. Descriptive statistics for all study variables per wave of data collection are provided in Table 2. A considerable portion of the participants (min: $31 \%$, max: $49 \%$ ) reported elevated levels of depression symptoms according to established cutoffs (Beck et al., 1996; Steer \& Beck, 1997). Of note, 119 participants had an average BDI-II score of $\geq$ 14 over the course of the longitudinal follow-up and displayed chronically elevated symptom levels. The observed variability in each study variable indicates that the full range of change in repetitive negative thinking, positive reappraisal, perceived stress, and depression severity can be adequately studied. 


\section{Longitudinal trajectories of emotion regulation habits}

\section{Repetitive negative thinking}

Table 3 details the results of the tested growth curve models for repetitive negative thinking. The ICC produced by the unconditional mean model (Model 1) showed that $70.78 \%$ of the total variation in repetitive negative thinking was between-person variance $(29.22 \%$ was within-person variation). The likelihood ratio tests showed that the adding the linear function in Model 2 improved the model fit of the unconditional mean model, $\chi^{2}(4)=485.92, p<.001$. Both growth parameters were significant for this unconditional linear growth model. The initial status of repetitive negative thinking was significantly different from zero $(p<.001)$, with an average level of 37.96 at the first wave of data collection. The significant linear effect $(p<.001)$ suggests that the average trajectory of repetitive negative thinking decreased each week with 0.394 points (scale: 0 to 108). Of note, there were significant individual differences around the prototypical change trajectory with respect to the intercept $S D=22.81$ (95\%-CI: 20.96, 24.83) and the linear slope $S D=0.72(95 \%$-CI: $0.61,0.85)$. Figure 1a depicts the prototypical (thick line) and individual (thin lines) trajectories of repetitive negative thinking.

Examining the contribution of depressive symptom severity and perceived stress at each wave in predicting repetitive negative thinking at the same wave, it was found that depressive symptom severity $(p<.001)$ and perceived stress $(p<.001)$ were significantly associated with repetitive negative thinking. As expected, higher levels of depressive symptoms and perceived stress were related to greater habitual use of repetitive negative thinking at the same wave, regardless of the linear trend in the habitual use of this emotion regulation strategy. Adding the time-varying covariates further improved the fit of Model 2, $\chi^{2}(2)=2506.44, p<.001$.

Finally, the results for the conditional linear growth model with age as a time-invariant covariate (Model 4) revealed that age was related to the initial levels of repetitive negative 
thinking $(p=.045)$, but not to the rate of linear change over time $(p=.805)$. However, adding age as a time-invariant covariate did not improve the fit of $\operatorname{Model} 3, \chi^{2}(2)=4.493, p=.106$.

\section{Positive reappraisal}

The results of the growth curve models tested for positive reappraisal are provided in Table 4. The ICC of the unconditional mean model (Model 1) suggested that $71.61 \%$ of the total variation in positive reappraisal scores can be explained by between-person variance (and $28.39 \%$ by within-person variation). The unconditional linear growth model (Model 2) had a better fit with the data than Model $1, \chi^{2}(4)=315.25, p<.001$. In this model, both the intercept $(p<.001)$ and linear slope $(p=.029)$ parameters were significant. Their unstandardized coefficients suggest that the average level of positive reappraisal at Wave 1 (10.24) was different from zero and linearly decreased by 0.02 points each week of data collection (scale: 0 to 14$)$. The variance in the intercepts $S D=3.49$ (95\%-CI: $3.20 ; 3.81)$ and linear slopes $S D=0.12$ (95\%-CI: $0.11 ; 0.14)$ was significant. The prototypical (thick line) and individual (thin lines) change trajectories for positive reappraisal are plotted in Figure $1 \mathrm{~b}$.

Examining within-person sources of individual differences in Model 3, the analyses showed that both depression severity $(p<.001)$ and perceived stress $(p<.001)$ levels were related to positive reappraisal at the same wave of data collection. Higher levels of depression severity

and perceived were related to lower habitual use of positive reappraisal within the same wave. Adding these time-varying covariates to Model 2 improved its fit, $\chi^{2}(2)=160.31, p<.001$.

Finally, the results for the conditional linear growth model with age as a time-invariant covariate (Model 4) showed that age was not related to the initial status of positive reappraisal $(p=.588)$, but may be a source of differential trajectories in positive reappraisal $(p=.019)$. Yet, adding age as a time-invariant covariate to Model 3 yielded only a marginal improvement of its fit with the data, $\chi^{2}(2)=5.81, p=.055$. 


\section{Depressive symptom severity and perceived stress}

Providing a benchmark to interpret change in emotion regulation habits, an unconditional mean and unconditional linear growth model were estimated for depressive symptom severity and perceived stress. Table 5 details the results of the growth models for depressive symptom severity and perceived stress. The ICCs produced by the unconditional mean models showed that $72.14 \%$ of the variation in perceived stress and $82.52 \%$ of the total variation in depressive symptom severity was between-person variance. Likelihood ratio tests demonstrated that the adding the linear function to the unconditional mean model for perceived stress, $\chi^{2}(4)=395.25, p<.001$, and for depressive symptom severity, $\chi^{2}(4)=570.14, p<.001$, improved the model fits. For perceived stress, the initial status differed from zero $(p<.001)$, with an average level of 16.73 at wave 1 . The linear effect $(p<.001)$ suggests that the average trajectory of perceived stress decreased each week with 0.07 points (scale: 0 to 40 ). For depressive symptom severity, the initial status was significantly different from zero $(p<.001)$, with an average level of 13.43 at the first wave of data collection. As for perceived stress, the significant linear effect $(p<.001)$ suggests that the average trajectory of depressive symptom severity decreased each week with 0.13 points (scale: 0 to 63 ). For both depressive symptom severity and perceived stress, there was significant variability in the initial status and linear change over time (see Table 5).

\section{Mechanisms of change in emotion regulation habits}

\section{Repetitive negative thinking}

In the model with perceived stress, the autocorrelations for both perceived stress, $b=0.156, S E=0.017, p<.001$, and repetitive negative thinking, $b=0.141, S E=.017, p<.001$, reached the .05 threshold for statistical significance. The cross-lagged relations showed that repetitive negative thinking predicted increases in perceived stress, $b=0.013, S E=0.005, p=.012$, 
and perceived stress predicted increases in repetitive negative thinking, $b=0.209, S E=0.054$, $p<.001$

In the model with depressive symptom severity, the autocorrelations for both depressive symptoms, $b=0.184, S E=0.016, p<.001$, and repetitive negative thinking, $b=0.158, S E=.016$, $p<.001$, were significant. As expected, repetitive negative thinking predicted increases in depressive symptoms, $b=0.019, S E=0.006, p<.001$, and depressive symptoms predicted increases in repetitive negative thinking, $b=0.092, S E=0.045, p=.040$.

\section{Positive reappraisal}

The model with habitual positive reappraisal showed significant autocorrelations for positive reappraisal, $b=0.101, S E=.014, p<.001$, and perceived stress, $b=0.178, S E=0.014$, $p<.001$. However, positive reappraisal did not predict changes in perceived stress, $b=-0.013$, $S E=0.025, p=.601$, and perceived stress was not related to subsequent changes in positive reappraisal, $b=-0.008, S E=0.007, p=.280$.

Similarly, in the model with depressive symptom severity, there were significant autocorrelations for both depressive symptoms, $b=0.210, S E=0.014, p<.001$, and positive reappraisal, $b=0.105, S E=.014, p<.001$. Yet, the results indicated that positive reappraisal was not related to increases in depressive symptoms, $b=-0.018, S E=0.028, p=.517$, and depressive symptoms were not related to increases in positive reappraisal, $b=0.005, S E=0.007, p=.474$.

\section{Discussion}

Investigating individual longitudinal trajectories of habitual repetitive negative thinking and positive reappraisal in adults, this study revealed that the prototypical patterns of change of both emotion regulation habits featured a linear decrease over the course of twenty weeks. Of note, perceived stress and depressive symptoms as benchmarks also exhibited linear decreases over time. However, the average linear decline in each of the study variables was weak, suggesting that the prototypical change trajectory of habitual repetitive negative thinking and 
positive reappraisal in adults may be relatively stable over time. Thus, when considering the average course of emotion regulation habits over time, this study provides support for views on emotion regulation habits as stable tendencies or "habits of thought" that occur frequently and repetitively in response to stress (Hertel, 2004; Nolen-Hoeksema et al., 2008; Smith \& Alloy, 2009; Watkins \& Nolen-Hoeksema, 2014). This observation in an adult sample adds to the few longitudinal studies examining the stability of habitual rumination. This previous work has reported both increasing (Hankin, 2008) and decreasing (Mazzer et al., 2019) trends in habitual rumination during adolescence as well as slightly declining levels in adults (Struijs et al., 2020). From a developmental perspective (cf. Hankin, 2008), these findings collectively suggest that the tendency to respond to distress with repetitive negative thinking may still be changing during adolescence and only crystalize into relatively stable characteristics when entering adulthood. In addition, this is the first to report on the longitudinal trajectory of positive reappraisal. While the prototypical course of habitual positive reappraisal use may be stable in adults, it remains unclear to what extent this emotion regulation tendency depends on developmental processes. More longitudinal research is needed that tracks individuals during transitions from adolescence to adulthood to uncover when these emotion regulation habits emerge and stabilize.

Importantly, this study observed that longitudinal trajectories of both repetitive negative thinking and positive reappraisal habits were not homogenous. Considerable individual variability occurred in the initial levels of emotion regulation habits as well as in the rate of change over time. This finding suggests that the propensity to use repetitive negative thinking or positive reappraisal is stable in some adults, but increases or decreases to varying degrees in others. Thus, habitual repetitive negative thinking and positive reappraisal may not have enduring properties in each individual. Exploring within-person sources of the variability in longitudinal trajectories, this study observed that higher levels of depressive symptoms and 
perceived stress during the past week are related to instantaneous increases in repetitive negative thinking and decreases in positive reappraisal during the same week. Both depressive symptom severity and perceived stress may concurrently modulate the habitual use of emotion regulation strategies, and potentially account for the decreasing longitudinal trend.

Examining the temporal precedence among these variables, within-person bidirectional relations with perceived stress and depressive symptom severity were evaluated. In line with transactional models of stress and vulnerability to depression (Hankin \& Abramson, 2001; Watkins \& Roberts, 2020), the results showed that repetitive negative thinking is dynamically related to perceived stress and depressive symptoms over time. In particular, perceived stress and depressive symptoms predicted increases in repetitive negative thinking, which in turn predicted subsequent increases in both perceived stress and depressive symptoms. This finding suggests that the degree of stability and change in an individual's tendency to use repetitive negative thinking depends on his/her perception of contextual features such as stressful circumstances and experienced depressive symptoms. An individual's propensity to engage in repetitive negative thinking may be particularly elevated when facing increases in experienced stress and symptoms of depression (Hertel, 2004; Watkins \& Nolen-Hoeksema, 2014). Interestingly, individuals with a greater tendency to engage in repetitive negative thinking may in turn elevate their perception of stress and depression levels. Thus, repetitive negative thinking may not only be a response to but also elicit higher levels of stress and increase depressive symptom severity. The finding of interlocking repetitive negative thinking and both perceived stress and symptoms of depression is consistent with recent research showing bidirectional relations between rumination and depressive symptoms in a single study (Whisman et al., 2020) and extends prior work examining these mutual relations with stress in separate studies (Flynn et al., 2010; Mazzer et al., 2019; Michl et al., 2013). Importantly, prior to this study the relations between repetitive negative thinking and perceived stress across time 
had not been studied systematically at the within-person level. However, this knowledge is important to verify theoretical predictions and identify modifiable targets for intervention.

In contrast to predictions derived from transactional models (Hankin \& Abramson, 2001; Watkins \& Roberts, 2020), the propensity to use positive reappraisal had no bidirectional relations with perceived stress or depressive symptoms. This study's observations suggest that the habitual use of positive reappraisal is not an antecedent or consequence of perceived stress or depressive symptom severity, but may reflect a correlate of concurrently experienced perceived stress or depressive symptom levers. Yet, this pattern of findings is surprising in light of research suggesting that positive reappraisal use is linked to prospective decreases in depressive symptoms (Brewer et al., 2016; Garnefski \& Kraaij, 2007; Haga et al., 2012), better stress recovery (Jamieson et al., 2012), and reduced daily life stress (Denny \& Ochsner, 2014). It is to note that the absence of bidirectional relations does not imply that habitual positive reappraisal it is unimportant in psychopathology. It is possible that the habitual use of positive reappraisal facilitates both adaptation and maladaptation depending on contextual factors. For example, prior research has found that state reappraisal use could be more adaptive when it is applied in uncontrollable as opposed to controllable situations (Haines et al., 2016; Troy, Shallcross, \& Mauss, 2013). Taking such appraisals of the broader context (e.g., chronic stressors or stressful periods in life) into account may be important to reveal when changes in habitual positive reappraisal may be associated with increases or decreases in perceived stress.

Several limitations to this study point to future directions. First, by conducting a twentywave follow-up over the course of twenty weeks, this longitudinal study retained a relatively high temporal resolution but covered only a period of five months. Caution is warranted in generalizing the observed trajectories of change to longer time periods. Future research could cover longer periods and critical transition phases (e.g., the transition from high school to college). Second, one out of four participants did not complete all twenty waves of data 
collection. Though overall compliance was relatively high (participants completed on average 17 of the 20 waves) and comparable to other research employing intensive longitudinal designs (Gruber, Kogan, Mennin, \& Murray, 2013), future research in MTurk samples should implement strategies to reduce attrition when conducting studies with similar sampling schemes (e.g., Hofmans, De Clercq, Kuppens, Verbeke, \& Widiger, 2019). Third, this study relied on self-report measures. Though all measures showed good internal consistency and reliability to detect within-subject change over time in this study), it is possible that these measures do not provide a complete picture of emotion regulation habits, perceived stress, and depression severity. Future research could integrate multiple methods of habitual emotion regulation (e.g., self and other reports) and psychopathology (e.g., self and clinician ratings) in longitudinal designs. Fourth, the general population sample from this study may limit the generalizability of the findings to clinical populations. Though this study was able to investigate emotion regulation habits along continuum broad range of depressive symptom and perceived stress levels, future work should replicate the findings in clinical samples to document the stability and change at clinical severity. Doing so, studies should assess whether participants receive any treatments to assess its impact on the course of emotion regulation habits. Fifth, the sample consisted mostly of persons who identified as female or described themselves as White, which limits the generalizability of the findings to other demographic groups. In light of prior research showing gender and racial/ethnic disparities in the occurrence of mood disorders (Breslau, Kendler, Su, Gaxiola-Aguilar, \& Kessler, 2005), future research should investigate whether the current findings about the role of repetitive negative thinking and positive reappraisal in depression are consistent across demographic groups. ${ }^{3}$ Finally, this study is limited by its focus on two emotion regulation habits. Future research should investigate longitudinal trajectories

\footnotetext{
${ }^{3}$ Exploring gender differences in this study, post hoc analyses revealed no differences in the use of repetitive negative thinking or positive reappraisal between women and men in the present study (see supplement 3 ).
} 
of other emotion regulation strategies (e.g., suppression, dampening) as well as their bidirectional relations with perceived stress and depressive symptoms.

Despite these limitations, this study augments knowledge of emotion regulation habits related to depression. The results showed that the prototypical individual change trajectories of habitual repetitive negative thinking and positive reappraisal were relatively stable over time, though considerable individual differences emerged in both the initial levels and rate of change. Explaining (some) of this variation, it was found that higher perceived stress and depressive symptom severity were related higher concurrent levels of habitual repetitive negative thinking and lower levels of positive reappraisal habits. Examining within-person processes of change, cross-lagged analysis revealed bidirectional relations between repetitive negative thinking and both perceived stress and depressive symptoms. No such within-person bidirectional relations were observed for positive reappraisal. Together, these findings help to gain a better understanding of the longitudinal stability and change in habitual repetitive negative thinking and positive reappraisal in adults. 


\section{References}

Aldao, A., Nolen-Hoeksema, S., \& Schweizer, S. (2010). Emotion-regulation strategies across psychopathology: A meta-analytic review. Clinical Psychology Review, 30(2), 217-237. https://doi.org/10.1016/j.cpr.2009.11.004

Beck, A. T., Epstein, N., Brown, G., \& Steer, R. A. (1988). An inventory for measuring clinical anxiety: Psychometric properties. Journal of Consulting and Clinical Psychology, 56(6), 893-897. https://doi.org/10.1037/0022-006X.56.6.893

Beck, A. T., Steer, R. A., \& Brown, G. K. (1996). Manual for the Beck Depression InventoryII. San Antonio, TX: Psychological Corporation.

Bolger, N., \& Laurenceau, J. P. (2013). Intensive longitudinal methods: An introduction to diary and experience sampling research. New York, NY: Guilford Press.

Brans, K., Koval, P., Verduyn, P., Lim, Y. L., \& Kuppens, P. (2013). The regulation of negative and positive affect in daily life. Emotion, 13(5), 926-939.

https://doi.org/10.1037/a0032400

Breslau, J., Kendler, K., Su, M., Gaxiola-Aguilar, S., \& Kessler, R. (2005). Lifetime risk and persistence of psychiatric disorders across ethnic groups in the United States. Psychological Medicine, 35(3), 317-327.

Brewer, S. K., Zahniser, E., \& Conley, C. S. (2016). Longitudinal impacts of emotion regulation on emerging adults: Variable- and person-centered approaches. Journal of Applied Developmental Psychology, 47, 1-12.

https://doi.org/10.1016/j.appdev.2016.09.002

Chandler, J., \& Shapiro, D. (2016). Conducting clinical research using crowdsourced convenience samples. Annual Review of Clinical Psychology, 12, 53-81.

https://doi.org/10.1146/annurev-clinpsy-021815-093623

Cohen, S., Kamarck, T., \& Mermelstein, R. (1983). A global measure of perceived stress. 
Journal of Health and Social Behavior, 24(4), 385-396.

Denny, B. T., \& Ochsner, K. N. (2014). Behavioral effects of longitudinal training in cognitive reappraisal. Emotion, 14(2), 425-433. https://doi.org/10.1037/a0035276

Ehring, T., Zetsche, U., Weidacker, K., Wahl, K., Schönfeld, S., \& Ehlers, A. (2011). The Perseverative Thinking Questionnaire (PTQ): Validation of a content-independent measure of repetitive negative thinking. Journal of Behavior Therapy and Experimental Psychiatry, 42(2), 225-232. https://doi.org/10.1016/j.jbtep.2010.12.003

Everaert, J., Grahek, I., Duyck, W., Buelens, J., Van den Bergh, N., \& Koster, E. H. W. (2017). Mapping the interplay among cognitive biases, emotion regulation, and depressive symptoms. Cognition and Emotion, 31(4), 726-735.

https://doi.org/10.1080/02699931.2016.1144561

Flynn, M., Kecmanovic, J., \& Alloy, L. B. (2010). An examination of integrated cognitiveinterpersonal vulnerability to depression: The role of rumination, perceived social support, and interpersonal stress generation. Cognitive Therapy and Research, 34(5), 456-466. https://doi.org/10.1007/s10608-010-9300-8

Garnefski, N., \& Kraaij, V. (2007). The Cognitive Emotion Regulation Questionnaire: Psychometric features and prospective relationships with depression and anxiety in adults. European Journal of Psychological Assessment, 23(3), 141-149.

https://doi.org/10.1027/1015-5759.23.3.141

Garnefski, N., Kraaij, V., \& Spinhoven, P. (2001). Negative life events, cognitive emotion regulation and emotional problems. Personality and Individual Differences, 30(8), 13111327. https://doi.org/10.1016/S0191-8869(00)00113-6

Graham, J. W. (2009). Missing Data Analysis: Making It Work in the Real World. Annual Review of Psychology, 60(1), 549-576. https://doi.org/10.1146/annurev.psych.58.110405.085530 
Gross, J. J., \& Jazaieri, H. (2014). Emotion, Emotion Regulation, and Psychopathology. Clinical Psychological Science, 2(4), 387-401. https://doi.org/10.1177/2167702614536164

Gross, J. J., \& John, O. P. (2003). Individual differences in two emotion regulation processes: Implications for affect, relationships, and well-being. Journal of Personality and Social Psychology, 85(2), 348-362.

Gruber, J., Kogan, A., Mennin, D., \& Murray, G. (2013). Real-world emotion? An experience-sampling approach to emotion experience and regulation in bipolar I disorder. Journal of Abnormal Psychology, 122(4), 971-983. https://doi.org/10.1037/a0034425

Haga, S. M., Ulleberg, P., Slinning, K., Kraft, P., Steen, T. B., \& Staff, A. (2012). A longitudinal study of postpartum depressive symptoms: multilevel growth curve analyses of emotion regulation strategies, breastfeeding self-efficacy, and social support. Archives of Women's Mental Health, 15(3), 1088-1094. https://doi.org/10.1007/s00737-0120274-2

Haines, S. J., Gleeson, J., Kuppens, P., Hollenstein, T., Ciarrochi, J., Labuschagne, I., ... Koval, P. (2016). The wisdom to know the difference: Strategy-situation fit in emotion regulation in daily life is associated with well-being. Psychological Science, 27(12), 1651-1659. https://doi.org/10.1177/0956797616669086

Hamaker, E. L., Kuiper, R. M., \& Grasman, R. P. P. P. (2015). A critique of the cross-lagged panel model. Psychological Methods, 20(1), 102-116. https://doi.org/10.1037/a0038889

Hankin, B. L. (2008). Stability of cognitive vulnerabilities to depression: A short-term prospective multiwave study. Journal of Abnormal Psychology, 117(2), 324-333. https://doi.org/10.1037/0021-843X.117.2.324

Hankin, B. L., \& Abramson, L. Y. (2001). Development of gender differences in depression: 
an elaborated cognitive vulnerability-transactional stress theory. Psychological Bulletin, 127(6), 773-796. Retrieved from http://www.ncbi.nlm.nih.gov/pubmed/11726071

Hertel, P. T. (2004). Memory for emotional and nonemotional events in depression: A question of habit? In D. Reisberg \& P. Hertel (Eds.), Memory and Emotion. New York: Oxford University Press.

Hofmans, J., De Clercq, B., Kuppens, P., Verbeke, L., \& Widiger, T. A. (2019, April 1). Testing the Structure and Process of Personality Using Ambulatory Assessment Data: An Overview of Within-Person and Person-Specific Techniques. Psychological Assessment. American Psychological Association Inc. https://doi.org/10.1037/pas0000562

Insel, T., Cuthbert, B., Garvey, M., Heinssen, R., Pine, D. S., Quinn, K., ... Wang, P. (2010). Research Domain Criteria (RDoC): Toward a new classification framework for research on mental disorders. American Journal of Psychiatry, 167(7), 748-751. https://doi.org/10.1176/appi.ajp.2010.09091379

Ireland, M. J., Clough, B. A., \& Day, J. J. (2017). The cognitive emotion regulation questionnaire: Factorial, convergent, and criterion validity analyses of the full and short versions. Personality and Individual Differences, 110, 90-95. https://doi.org/10.1016/j.paid.2017.01.035

Jamieson, J. P., Nock, M. K., \& Mendes, W. B. (2012). Mind over matter: Reappraising arousal improves cardiovascular and cognitive responses to stress. Journal of Experimental Psychology: General, 141(3), 417-422. https://doi.org/10.1037/a0025719

Joiner, T. E., Walker, R. L., Pettit, J. W., Perez, M., \& Cukrowicz, K. C. (2005). Evidencebased assessment of depression in adults. Psychological Assessment, 17(3), 267-277. https://doi.org/10.1037/1040-3590.17.3.267

Joormann, J. (2010). Cognitive inhibition and emotion regulation in depression. Current Directions in Psychological Science, 19(3), 161-166. 
https://doi.org/10.1177/0963721410370293

Joormann, J., Dkane, M., \& Gotlib, I. H. (2006). Adaptive and Maladaptive Components of Rumination? Diagnostic Specificity and Relation to Depressive Biases. Behavior Therapy, 37(3), 269-280. https://doi.org/10.1016/j.beth.2006.01.002

Liu, D. Y., \& Thompson, R. J. (2017). Selection and implementation of emotion regulation strategies in major depressive disorder: An integrative review. Clinical Psychology Review, 0. https://doi.org/https://doi.org/10.1016/j.cpr.2017.07.004

Mahoney, A. E. J., McEvoy, P. M., \& Moulds, M. L. (2012). Psychometric properties of the Repetitive Thinking Questionnaire in a clinical sample. Journal of Anxiety Disorders, 26(2), 359-367. https://doi.org/10.1016/j.janxdis.2011.12.003

Mazzer, K., Boersma, K., \& Linton, S. J. (2019). A longitudinal view of rumination, poor sleep and psychological distress in adolescents. Journal of Affective Disorders, 245, 686-696. https://doi.org/10.1016/J.JAD.2018.11.053

McEvoy, P. M., Mahoney, A. E. J., \& Moulds, M. L. (2010). Are worry, rumination, and post-event processing one and the same?: Development of the repetitive thinking questionnaire. Journal of Anxiety Disorders, 24(5), 509-519. https://doi.org/10.1016/j.janxdis.2010.03.008

McRae, K., Ciesielski, B., \& Gross, J. J. (2012). Unpacking cognitive reappraisal: Goals, tactics, and outcomes. Emotion, 12(2), 250-255. https://doi.org/10.1037/a0026351

Michl, L. C., McLaughlin, K. A., Shepherd, K., \& Nolen-Hoeksema, S. (2013). Rumination as a mechanism linking stressful life events to symptoms of depression and anxiety: longitudinal evidence in early adolescents and adults. Journal of Abnormal Psychology, 122(2), 339-352. https://doi.org/10.1037/a0031994

Nezlek, J. B., \& Kuppens, P. (2008). Regulating Positive and Negative Emotions in Daily Life. Journal of Personality, 76(3), 561-580. https://doi.org/10.1111/j.1467- 
6494.2008.00496.x

Nolen-Hoeksema, S., Wisco, B. E., \& Lyubomirsky, S. (2008). Rethinking rumination. Perspectives on Psychological Science, 3(5), 400-424. https://doi.org/10.1111/j.17456924.2008.00088.x

Ochsner, K. N., \& Gross, J. J. (2008). Cognitive emotion regulation: Insights from social cognitive and affective neuroscience. Current Directions in Psychological Science, 17(2), 153-158. https://doi.org/10.1111/j.1467-8721.2008.00566.x

Ophir, Y., Sisso, I., Asterhan, C. S. C., Tikochinski, R., \& Reichart, R. (2018). The Turker Blues: Hidden Factors Behind Increased Depression Rates Among Amazon's Mechanical Turkers. https://doi.org/10.1177/2167702619865973

Pbert, L., Doerfler, L. A., \& DeCosimo, D. (1992). An evaluation of the perceived stress scale in two clinical populations. Journal of Psychopathology and Behavioral Assessment, 14(4), 363-375. https://doi.org/10.1007/BF00960780

Pinheiro, J., Bates, D., DebRoy, S., Sarkar, D., \& Team, R. C. (2018). nlme: Linear and Nonlinear Mixed Effects Models. Retrieved from https://cran.rproject.org $/$ package $=$ nlme

R Core Team. (2018). R: A language and environment for statistical computing. Vienna, Austria: R Foundation for Statistical Computing. Retrieved from www.R-project.org Raes, F. (2012). Repetitive Negative Thinking Predicts Depressed Mood at 3-Year Follow-up in Students. Journal of Psychopathology and Behavioral Assessment, 34(4), 497-501. https://doi.org/10.1007/s10862-012-9295-4

Roberti, J. W., Harrington, L. N., \& Storch, E. A. (2006). Further Psychometric Support for the 10-Item Version of the Perceived Stress Scale. Journal of College Counseling, 9(2), 135-147. https://doi.org/10.1002/j.2161-1882.2006.tb00100.x

Singer, J. D., \& Willett, J. B. (2003). Applied longitudinal data analysis: Modeling change 
and event pccurrence. New York: Oxford University Press.

Smith, J. M., \& Alloy, L. B. (2009). A roadmap to rumination: a review of the definition, assessment, and conceptualization of this multifaceted construct. Clinical Psychology Review, 29(2), 116-128. https://doi.org/10.1016/j.cpr.2008.10.003

Spinhoven, P., van Hemert, A. M., \& Penninx, B. W. (2018). Repetitive negative thinking as a predictor of depression and anxiety: A longitudinal cohort study. Journal of Affective Disorders, 241, 216-225. https://doi.org/10.1016/j.jad.2018.08.037

Steer, R. A., \& Beck, A. T. (1997). Beck Anxiety Inventory. In C. P. Zalaquett \& R. J. Wood (Eds.), Evaluating stress: A book of resources (pp. 23-40). Lanham, HD: Scarecrow Education.

Struijs, S. Y., Lamers, F., Verdam, M. G. E., van Ballegooijen, W., Spinhoven, P., van der Does, W., \& Penninx, B. W. J. H. (2020). Temporal stability of symptoms of affective disorders, cognitive vulnerability and personality over time. Journal of Affective Disorders, 260, 77-83. https://doi.org/10.1016/j.jad.2019.08.090

Topper, M., Molenaar, D., Emmelkamp, P. M. G., \& Ehring, T. (2014). Are Rumination and Worry Two Sides of the Same Coin? A Structural Equation Modelling Approach. Journal of Experimental Psychopathology, 5(3), jep.038813. https://doi.org/10.5127/jep.038813

Troy, A. S., Shallcross, A. J., \& Mauss, I. B. (2013). A Person-by-Situation Approach to Emotion Regulation. Psychological Science, 24(12), 2505-2514. https://doi.org/10.1177/0956797613496434

Watkins, E. R. (2008). Constructive and unconstructive repetitive thought. Psychological Bulletin, 134(2), 163-206. https://doi.org/10.1037/0033-2909.134.2.163

Watkins, E. R., \& Nolen-Hoeksema, S. (2014). A habit-goal framework of depressive rumination. Journal of Abnormal Psychology, 123(1), 24-34. 
https://doi.org/10.1037/a0035540

Watkins, E. R., \& Roberts, H. (2020). Reflecting on rumination: Consequences, causes, mechanisms and treatment of rumination. Behaviour Research and Therapy, 103573. https://doi.org/10.1016/j.brat.2020.103573

Waugh, C. E., Shing, E. Z., Avery, B. M., Jung, Y., Whitlow, C. T., \& Maldjian, J. A. (2017). Neural predictors of emotional inertia in daily life. Social Cognitive and Affective Neuroscience, 12(9), 1448-1459. https://doi.org/10.1093/scan/nsx071

Whisman, M. A., du Pont, A., \& Butterworth, P. (2020). Longitudinal associations between rumination and depressive symptoms in a probability sample of adults. Journal of Affective Disorders, 260, 680-686. https://doi.org/10.1016/j.jad.2019.09.035 
Table 1. Demographic characteristics.

\begin{tabular}{lc}
\hline Age $(M)$ & $39.25(S D=12.30)$ \\
Gender $(\%)$ & \\
Male & 27.81 \\
Female & 72.19 \\
Race $(\%)$ & 79.69 \\
White or Caucasian & 8.44 \\
Black or African American & 6.25 \\
Asian American & 4.06 \\
Hispanic American & 1.56 \\
Other & \\
Education $(\%)$ & 0.63 \\
Some high school & 8.13 \\
High school graduate & 27.19 \\
Some college & 10.31 \\
Two-year college graduate & 35.31 \\
Four-year college graduate & 14.69 \\
Master degree & 3.75 \\
Graduate or professional degree & \\
\hline
\end{tabular}


Table 2. Descriptive statistics for study variables per wave of data collection.

\begin{tabular}{|c|c|c|c|c|c|c|c|c|c|c|c|c|c|c|c|c|c|}
\hline \multirow[t]{2}{*}{ wave } & \multirow[t]{2}{*}{$N$} & \multicolumn{4}{|c|}{ Depressive symptoms } & \multicolumn{4}{|c|}{$\begin{array}{l}\text { Repetitive negative } \\
\text { thinking }\end{array}$} & \multicolumn{4}{|c|}{ Positive reappraisal } & \multicolumn{4}{|c|}{ Perceived stress } \\
\hline & & $M$ & $S D$ & Min & Max & $M$ & $S D$ & Min & Max & $M$ & $S D$ & Min & Max & $M$ & $S D$ & Min & Max \\
\hline 0 & 320 & 14.88 & 12.18 & 0 & 58 & 69.68 & 27.17 & 27 & 135 & 14.14 & 4.17 & 4 & 20 & 18.18 & 8.17 & 0 & 40 \\
\hline 1 & 320 & 13.30 & 11.74 & 0 & 54 & 66.91 & 26.32 & 27 & 135 & 14.33 & 4.11 & 4 & 20 & 16.87 & 8.39 & 0 & 40 \\
\hline 2 & 320 & 12.95 & 12.56 & 0 & 55 & 63.40 & 26.24 & 27 & 131 & 14.54 & 4.31 & 4 & 20 & 16.22 & 8.55 & 0 & 40 \\
\hline 3 & 320 & 12.92 & 13.09 & 0 & 57 & 63.24 & 27.00 & 27 & 134 & 13.95 & 4.38 & 4 & 20 & 16.43 & 8.60 & 0 & 40 \\
\hline 4 & 314 & 12.15 & 12.48 & 0 & 56 & 61.08 & 27.50 & 27 & 134 & 14.20 & 4.55 & 4 & 20 & 15.73 & 8.55 & 0 & 39 \\
\hline 5 & 297 & 11.94 & 12.74 & 0 & 61 & 61.51 & 27.82 & 27 & 135 & 14.18 & 4.65 & 4 & 20 & 15.57 & 8.68 & 0 & 40 \\
\hline 6 & 290 & 12.02 & 12.67 & 0 & 58 & 60.16 & 27.63 & 27 & 131 & 14.03 & 4.76 & 4 & 2 & 5.51 & .49 & $\sigma$ & 40 \\
\hline 7 & 281 & 11.90 & 12.41 & 0 & 61 & 60.93 & 27.81 & 27 & 129 & 14.03 & 4.89 & 4 & 20 & 15.54 & 8.84 & 0 & 40 \\
\hline 8 & 269 & 11.58 & 12.47 & 0 & 60 & 60.76 & 28.36 & 27 & 13 & 13.95 & 4.59 & 4 & 2 & 15.93 & 8.75 & 0 & 40 \\
\hline 9 & 267 & 11.85 & 12.45 & 0 & 58 & 60.44 & 27.97 & 27 & 135 & 14.00 & 4.86 & 4 & 20 & 15.81 & 9.18 & 0 & 40 \\
\hline 10 & 264 & 11.61 & 12.50 & 0 & 58 & 61.79 & 28.89 & 27 & 132 & 13.86 & 4.89 & 4 & 2 & 15.76 & 8.87 & 0 & 40 \\
\hline 11 & 261 & 11.55 & 12.86 & 0 & 59 & 59.82 & 28.42 & 27 & 132 & 13.84 & 4.77 & 4 & 20 & 15.57 & .93 & 0 & 40 \\
\hline 12 & 257 & 11.00 & 12.47 & 0 & 56 & 58.15 & 26.55 & 27 & 131 & 13.88 & 4.95 & 4 & 20 & 15.38 & 8.54 & 0 & 40 \\
\hline 13 & 252 & 10.49 & 12.26 & 0 & 56 & 57.64 & 27.26 & 27 & 131 & 13.81 & 4.86 & 4 & 20 & 15.10 & 8.52 & 0 & 39 \\
\hline 14 & 250 & 11.01 & 12.60 & 0 & 57 & 59.75 & 28.61 & 27 & 135 & 13.55 & 5.21 & 4 & 20 & 15.57 & 9.29 & 0 & 40 \\
\hline 15 & 246 & 10.54 & 12.53 & 0 & 55 & 57.22 & 28.31 & 27 & 133 & 13.94 & 4.97 & 4 & 20 & 14.69 & 9.02 & 0 & 38 \\
\hline 16 & 244 & 10.16 & 12.16 & 0 & 55 & 56.66 & 27.25 & 27 & 131 & 13.66 & 5.22 & 4 & 20 & 14.60 & 8.63 & 0 & 40 \\
\hline 17 & 242 & 10.38 & 12.19 & 0 & 58 & 58.96 & 28.08 & 27 & 135 & 13.70 & 5.09 & 4 & 20 & 15.02 & 8.88 & 0 & 40 \\
\hline 18 & 240 & 10.76 & 12.64 & 0 & 59 & 58.56 & 27.81 & 27 & 132 & 13.71 & 5.05 & 4 & 20 & 15.45 & 9.17 & 0 & 39 \\
\hline 19 & 240 & 10.96 & 12.89 & 0 & 60 & 60.78 & 29.92 & 27 & 135 & 13.75 & 5.08 & 4 & 20 & 15.41 & 9.16 & 0 & 40 \\
\hline
\end{tabular}

Notes. The questionnaire scores were not rescaled in this table. For all fitted multilevel models, the total scores were rescaled so that the minimum possible value equaled 0 . 
Table 3. Fitted individual growth curve models for repetitive negative thinking.

\begin{tabular}{|c|c|c|c|c|c|c|c|c|c|c|c|c|}
\hline \multirow[t]{3}{*}{ Model } & \multicolumn{6}{|c|}{ Fixed effects } & \multicolumn{6}{|l|}{ Random effects } \\
\hline & \multirow{2}{*}{\multicolumn{2}{|c|}{ Effect }} & \multirow{2}{*}{ Coef. } & \multirow{2}{*}{$S E$} & \multirow{2}{*}{$t$} & \multirow{2}{*}{$p$} & \multirow{2}{*}{ Effect } & \multirow{2}{*}{\multicolumn{2}{|c|}{ Estimate }} & \multirow{2}{*}{$S D$} & \multicolumn{2}{|c|}{$\mathrm{CI}_{95}(S D)$} \\
\hline & & & & & & & & & & & lower & upper \\
\hline \multicolumn{13}{|r|}{$-P P=$} \\
\hline & \multirow{2}{*}{\multicolumn{2}{|c|}{ Intercept }} & 34.712 & 1.332 & 26.070 & $<0.001$ & Intercept & & 550.986 & 23.473 & 21.673 & 25.422 \\
\hline & & & & & & & Residual & & 227.435 & 15.081 & 14.793 & 15.374 \\
\hline \multicolumn{13}{|l|}{2} \\
\hline \multirow{2}{*}{\multicolumn{2}{|c|}{$\begin{array}{l}\text { Intercept } \\
\text { Wave }\end{array}$}} & 37.955 & 1.347 & 28.183 & $3<0.001$ & \multicolumn{2}{|c|}{1 Intercept } & 520.397 & & \multirow{2}{*}{$\begin{array}{c}20.956 \\
0.610\end{array}$} & \multicolumn{2}{|c|}{24.833} \\
\hline & & -0.394 & 0.061 & 6.453 & $<0.001$ & \multirow{2}{*}{\multicolumn{2}{|c|}{$\begin{array}{l}\text { Linear slope } \\
\text { Correlation intercept - wave }\end{array}$}} & 0.519 & 0.721 & & 0.852 & \\
\hline & & & & & & & & \multirow{2}{*}{$\begin{array}{l}-0.055 \\
212.74\end{array}$} & & -0.182 & \multicolumn{2}{|l|}{0.074} \\
\hline & & & & & & \multicolumn{2}{|c|}{ Residual } & & 14.586 & $6 \quad 14.262$ & 214.916 & \\
\hline & & & & & & Auto & correlation & 0.200 & & 0.167 & 0.232 & \\
\hline 3 & & & & & & & & & & & & \\
\hline & Interce & & 36.231 & 1.360 & 26.645 & $<0.001$ & Intercept & & 560.136 & 23.667 & 21.788 & 25.708 \\
\hline & Wave & & -0.188 & 0.0449 & -4.196 & $<0.001$ & Linear slope & & 0.288 & 0.536 & 0.455 & 0.633 \\
\hline & BDI & & 0.840 & 0.0360 & 23.312 & $<0.001$ & Correlation intercept $-v$ & rave & -0.124 & & -0.309 & 0.069 \\
\hline & PSS & & 1.346 & 0.0405 & 33.220 & $<0.001$ & Residual & & 125.884 & 11.220 & 10.985 & 11.459 \\
\hline & & & & & & & Autocorrelation & & 0.115 & & 0.083 & 0.147 \\
\hline 4 & & & & & & & & & & & & \\
\hline & Interce & & 36.228 & 1.351 & 26.807 & $<0.001$ & Intercept & & 552.703 & 23.510 & 21.664 & 25.513 \\
\hline & Wave & & -0.187 & 0.045 & 4.156 & $<0.001$ & Linear slope & & 0.288 & 0.536 & 0.455 & 0.633 \\
\hline & BDI & & 0.840 & 0.036 & 23.310 & $<0.001$ & Correlation intercept $-v$ & ave & -0.126 & & -0.284 & 0.038 \\
\hline & PSS & & 1.350 & 0.041 & 33.214 & $<0.001$ & Residual & & 125.879 & 11.220 & 10.985 & 11.459 \\
\hline & Age & & -0.221 & 0.110 & 2.009 & 0.045 & Autocorrelation & & 0.115 & & 0.083 & 0.147 \\
\hline & Wave & Age & -0.001 & 0.004 & 0.247 & 0.805 & & & & & & \\
\hline
\end{tabular}

Note. Model 1 (Deviance: 46583, AIC: 46589, BIC: 46609); Model 2 (Deviance: 46097, AIC: 46111, BIC: 46157); Model 3

(Deviance: 43591, AIC: 43609, BIC: 43668); Model 4 (Deviance: 43586, AIC: 43608, BIC: 43681). 
Table 4. Fitted individual growth curve models for positive reappraisal.

\begin{tabular}{|c|c|c|c|c|c|c|c|c|c|c|c|}
\hline \multirow[t]{3}{*}{ Model } & \multicolumn{5}{|l|}{ Fixed effects } & \multicolumn{6}{|l|}{ Random effects } \\
\hline & \multirow{2}{*}{ Effect } & \multirow{2}{*}{ Coef. } & \multirow{2}{*}{$S E$} & \multirow{2}{*}{$t$} & \multirow{2}{*}{$p$} & \multirow{2}{*}{\multicolumn{2}{|c|}{ Effect }} & \multirow{2}{*}{ Estimate } & \multirow{2}{*}{$S D$} & \multicolumn{2}{|c|}{$\mathrm{CI}_{95}(S D)$} \\
\hline & & & & & & & & & & lower & upper \\
\hline \multirow[t]{3}{*}{1} & & & & & & & & & & & \\
\hline & Intercept & 10.057 & 0.225 & 44.624 & $<.001$ & Intercept & & 15.803 & 3.975 & 3.671 & 4.304 \\
\hline & & & & & & Res & & 6.263 & 2.503 & 2.455 & 2.552 \\
\hline \multicolumn{12}{|l|}{2} \\
\hline Intercept & 10.243 & 0.206 & 49.702 & 0.000 & \multicolumn{2}{|l|}{ Intercept } & 12.172 & 3.489 & 3.199 & 3.805 & \\
\hline \multirow[t]{4}{*}{ Wave } & -0.022 & 0.010 & 2.185 & 0.029 & \multicolumn{2}{|c|}{ Linear slope } & 0.015 & 0.122 & 0.105 & 0.142 & \\
\hline & & & & & \multicolumn{2}{|c|}{ Correlation intercept - wave } & 0.345 & & 0.155 & 0.510 & \\
\hline & & & & & \multicolumn{2}{|c|}{ Residual } & 5.850 & 2.419 & 2.368 & 2.470 & \\
\hline & & & & & \multicolumn{2}{|c|}{ Autocorrelation } & 0.110 & & 0.078 & 0.141 & \\
\hline \multicolumn{12}{|c|}{ 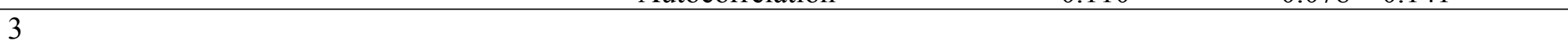 } \\
\hline & Intercept & 10.318 & 0.208 & 49.714 & $<0.001$ & \multicolumn{2}{|l|}{ Intercept } & 12.401 & 3.521 & 3.231 & 3.838 \\
\hline & Wave & -0.031 & 0.010 & -3.156 & 0.002 & \multicolumn{2}{|l|}{ Linear slope } & 0.015 & 0.121 & 0.104 & 0.141 \\
\hline & BDI & -0.032 & 0.008 & -4.201 & $<0.001$ & \multicolumn{2}{|c|}{ Correlation intercept - wave } & 0.314 & & 0.136 & 0.472 \\
\hline & \multirow[t]{2}{*}{ PSS } & -0.071 & 0.009 & -8.255 & $<0.001$ & \multirow{2}{*}{\multicolumn{2}{|c|}{$\begin{array}{l}\text { Residual } \\
\text { Autocorrelation }\end{array}$}} & 5.654 & 2.378 & 2.328 & 2.428 \\
\hline & & & & & & & & 0.105 & & 0.074 & 0.137 \\
\hline 4 & & & & & & & & & & & \\
\hline & Intercept & 10.322 & 0.208 & 49.744 & $<0.001$ & Inter & & 12.390 & 3.520 & 3.230 & 3.836 \\
\hline & Wave & -0.033 & 0.010 & 3.338 & $<0.001$ & Linear slope & & 0.014 & 0.119 & 0.102 & 0.139 \\
\hline & BDI & -0.032 & 0.008 & 4.242 & $<0.001$ & Correlation intercept & wave & 0.330 & & 0.151 & 0.488 \\
\hline & PSS & -0.071 & 0.009 & 8.270 & $<0.001$ & Residual & & 5.655 & 2.378 & 2.329 & 2.428 \\
\hline & Age & -0.009 & 0.017 & 0.543 & 0.588 & Autocorrelation & & 0.106 & & 0.074 & 0.137 \\
\hline & Wave * Age & 0.002 & 0.001 & 2.338 & 0.019 & & & & & & \\
\hline
\end{tabular}

Note. Model 1 (Deviance: 26861, AIC: 26867, BIC: 26887); Model 2 (Deviance: 26546, AIC: 26560, BIC: 26606); Model 3 (Deviance: 26386, AIC: 26404, BIC: 26463); Model 4 (Deviance: 26380, AIC: 26402, BIC: 26475). 
Table 5. Fitted individual growth curve models for depressive symptom severity and perceived stress.

\begin{tabular}{|c|c|c|c|c|c|c|c|c|c|c|}
\hline Model & Fixed eff & fects & & & & Random effects & & & & \\
\hline & Effect & Coef & SF & $t$ & $n$ & Effect & Fstimate & $S D$ & & $95(S D)$ \\
\hline & LIItect & . & ND & $t$ & $P$ & Lilect & Estminate & הע & lower & upper \\
\hline Perceived & d stress & & & & & & & & & \\
\hline Model $1^{\mathrm{a}}$ & Intercept & 16.10 & 0.424 & 37.960 & $<.001$ & Intercept & 56.021 & 7.485 & 6.912 & 8.105 \\
\hline & & & & & & Residual & 21.6311 & 4.651 & 4.562 & 4.741 \\
\hline Model $2^{b}$ & Intercept & 16.731 & 0.420 & 39.794 & $<.001$ & Intercept & 50.500 & 7.106 & 6.552 & 7.742 \\
\hline & Wave & -0.075 & 0.018 & -4.186 & $<.001$ & Linear slope & 0.036 & 0.190 & 0.155 & 0.233 \\
\hline & & & & & & Correlation intercept - wave & 0.078 & & -0.094 & 0.246 \\
\hline & & & & & & Residual & 20.997 & 4.582 & 4.480 & 4.687 \\
\hline & & & & & & Autocorrelation & 0.217 & & 0.184 & 0.248 \\
\hline Depressil & ve symptom & severity & & & & & & & & \\
\hline Model $1^{\mathrm{c}}$ & Intercept & 12.345 & 0.650 & 18.992 & $<.001$ & Intercept & 133.140 & 11.539 & 10.665 & 12.484 \\
\hline & & & & & & Residual & 28.205 & 5.311 & 2.509 & 5.414 \\
\hline Model $2^{\mathrm{d}}$ & Intercept & 13.428 & 0.644 & 20.854 & $<.001$ & Intercept & 124.748 & 11.169 & 10.230 & 12.115 \\
\hline & Wave & -0.131 & 0.023 & 5.817 & $<.001$ & Linear slope & 0.073 & 0.270 & 0.228 & 0.319 \\
\hline & & & & & & Correlation intercept - wave & 0.025 & & -0.055 & 0.105 \\
\hline & & & & & & Residual & 5.142 & & 5.024 & 5.262 \\
\hline & & & & & & Autocorrelation & 0.234 & & 0.201 & 0.266 \\
\hline
\end{tabular}

Note. Model 1=unconditional mean model; Model 2=unconditional linear growth model; ${ }^{\mathrm{a}}$ Deviance: 33678, AIC: 33684, BIC: $33704 ;{ }^{\mathrm{b}}$ Deviance: 33283, AIC: 33297, BIC: 33343; ${ }^{\mathrm{c}}$ Deviance: 35324, AIC: 35330, BIC: 35350; ${ }^{\mathrm{d}}$ Deviance: 34754, AIC: 34768 , BIC:34814. 
The authors declare that they have no conflict of interest. 


\section{Contributors}

Jonas Everaert (JE) and Jutta Joormann (JJ) developed the study concept and designed the study. Testing, data collection, and data analysis were performed by JE under the supervision of JJ. JE drafted the paper and JJ provided critical revisions. Both authors approved the final version of the paper for submission.

\section{Acknowledgements}

None.

\section{Role of the Funding Source}

This work was supported by grants from the Belgian American Educational Foundation, the Special Research Fund at Ghent University, and a postdoctoral fellowship from the Research Foundation - Flanders. The funders had no role in study design, data collection and analysis, decision to publish, or preparation of the manuscript.

\section{Data statement}

Anonymized code can be made available for the anonymous reviewers and will be made publicly available via the Open Science Framework upon acceptance of this manuscript. 


\section{Supplement 1: Data quality requirements.}

Following recommendations for research using crowdsourced samples (Chandler \& Shapiro, 2016), several steps were taken to ensure high data quality and power. Only MTurk workers with a history of providing good-quality responses (i.e., an acceptance ratio of $\geq 95 \%$ ) were allowed to participate. Moreover, three reading check questions were presented during the surveys to discriminate attentive from inattentive MTurk workers. For example, one validation question read: "Thank you for your work in this survey so far. To show that you are a human, please refuse to answer this question: How many fingers does a typical person have on each hand?". Respondents were then given four response options (e.g., five, six, ten, and three) which they had to leave blank. These questions were presented at irregular intervals and participants were required to correctly answer at least two questions. Participants received a notification when they failed to meet this criterion. Finally, in line with prior longitudinal studies in MTurk samples (Dejonckheere, Bastian, Fried, Murphy, \& Kuppens, 2017), an additional requirement for inclusion in the study was that participants met criteria for compliance with the study protocol. It was decided that participants had to complete at least four out of the twenty waves (i.e., 20\%) of data collection. With such data quality requirements, previous research has demonstrated that MTurk data are comparable to those collected in the laboratory (Chandler \& Shapiro, 2016). 


\section{Supplement 2: Questionnaire instructions.}

All participants were instructed to complete the questionnaires in this study in reference to the past week. This was to standardize the temporal orientation across all questionnaires and waves of data collection. The precise instructions for each questionnaire are detailed below.

Repetitive negative thinking subscale of the Repetitive Thinking Questionnaire (McEvoy, Mahoney, \& Moulds, 2010): “Please answer the following questions in relation to distressing or upsetting situations that happened to you last week (including today). How true are each of these statements with respect to your experience after the distressing or upsetting situations?".

Positive reappraisal subscale of the Cognitive Emotion Regulation Questionnaire (Garnefski et al., 2001): "Below is a list of statements that relate to things you might do when you experience negative emotions or unpleasant events. Think about the unpleasant events that happened last week and/or the negative emotions you had. Indicate how often you thought the following things during the last week, including today.".

Beck Depression Inventory - II (BDI-II; Beck et al., 1996): “Below are 21 groups of statements. For each group, pick out the one statement that bests describes the way you have been feeling during the past week, including today. If several statements seem to apply equally well, pick the highest number for that group."

Perceived Stress Scale (Cohen, Kamarck, \& Mermelstein, 1983): “The following questions will ask you about your feelings and thoughts during the past week, including today. Please indicate how often you felt or thought a certain way." 


\section{Supplement 3: Modeling the role of gender differences.}

Prior research has reported gender differences in emotion regulation with women engaging more in emotional support seeking, rumination, and positive self-talk (e.g., Tamres, Janicki, \& Helgeson, 2002). To explore the role of gender differences in this study, gender was added as a Level-2 predictor of the random intercepts of Model 2 (see the analytical plan in the main manuscript). The $\mathrm{R}$ outputs below indicate that no gender differences emerged in the use of repetitive negative thinking or positive reappraisal in the present study. However, these findings should be interpreted with caution because our study was not a priori designed to investigate gender differences, which resulted in a skewed distribution.

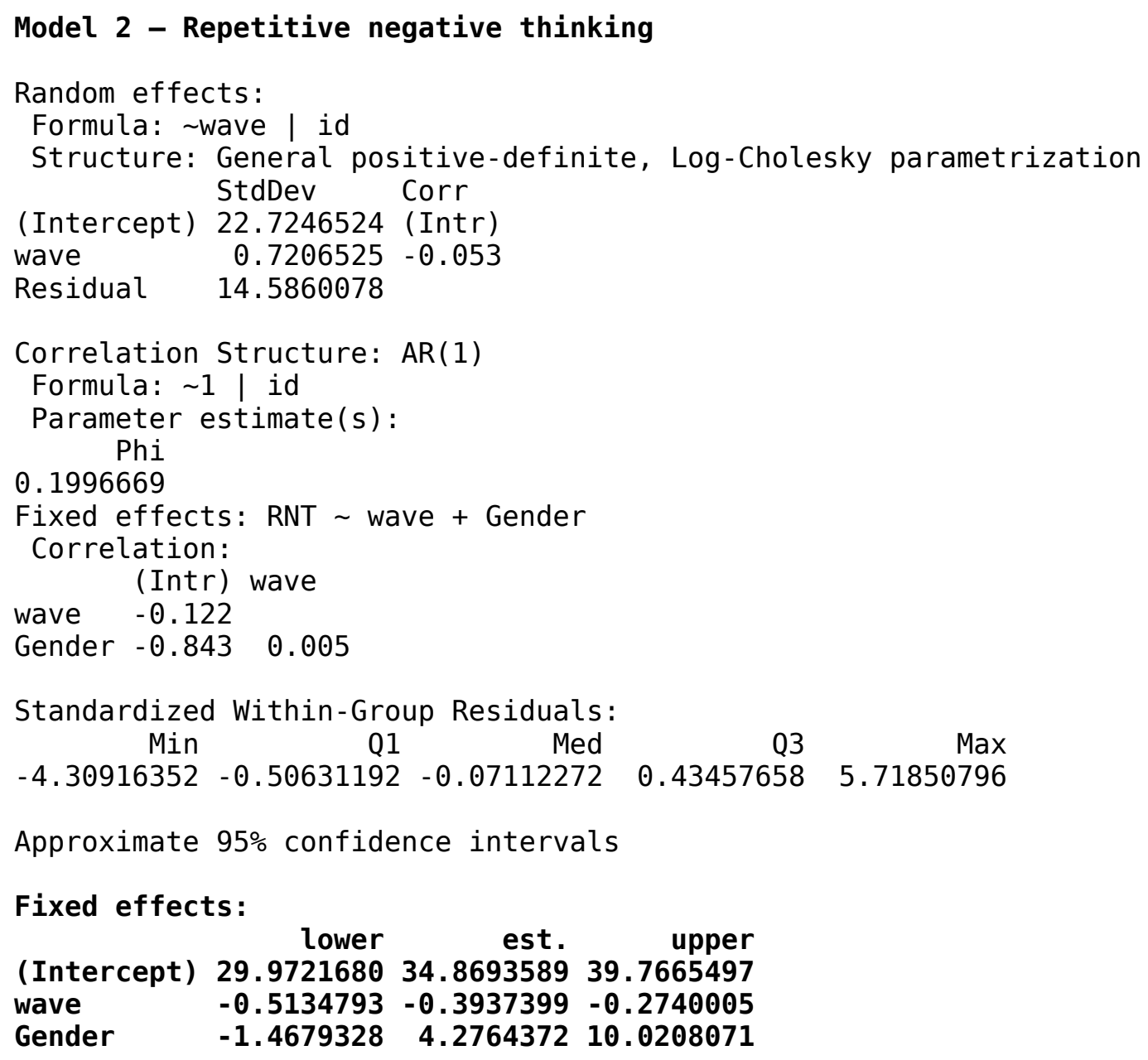


attr (, "label")

[1] "Fixed effects:"

Random Effects:

Level: id

$\begin{array}{lrrr}\mathrm{sd}((\text { Intercept ) } & 20.8861929 & 22.72465243 & 24.72493823 \\ \mathrm{sd}(\text { wave) } & 0.6116570 & 0.72065248 & 0.84907069 \\ \text { cor((Intercept), wave) } & -0.1600433 & -0.05336794 & 0.05453956\end{array}$

Correlation structure:

$$
\text { lower est. upper }
$$

Phi $0.1670953 \quad 0.19966690 .2318031$

attr (, "label")

[1] "Correlation structure:"

Within-group standard error:

$$
\text { lower est. upper }
$$

$14.26357 \quad 14.58601 \quad 14.91573$

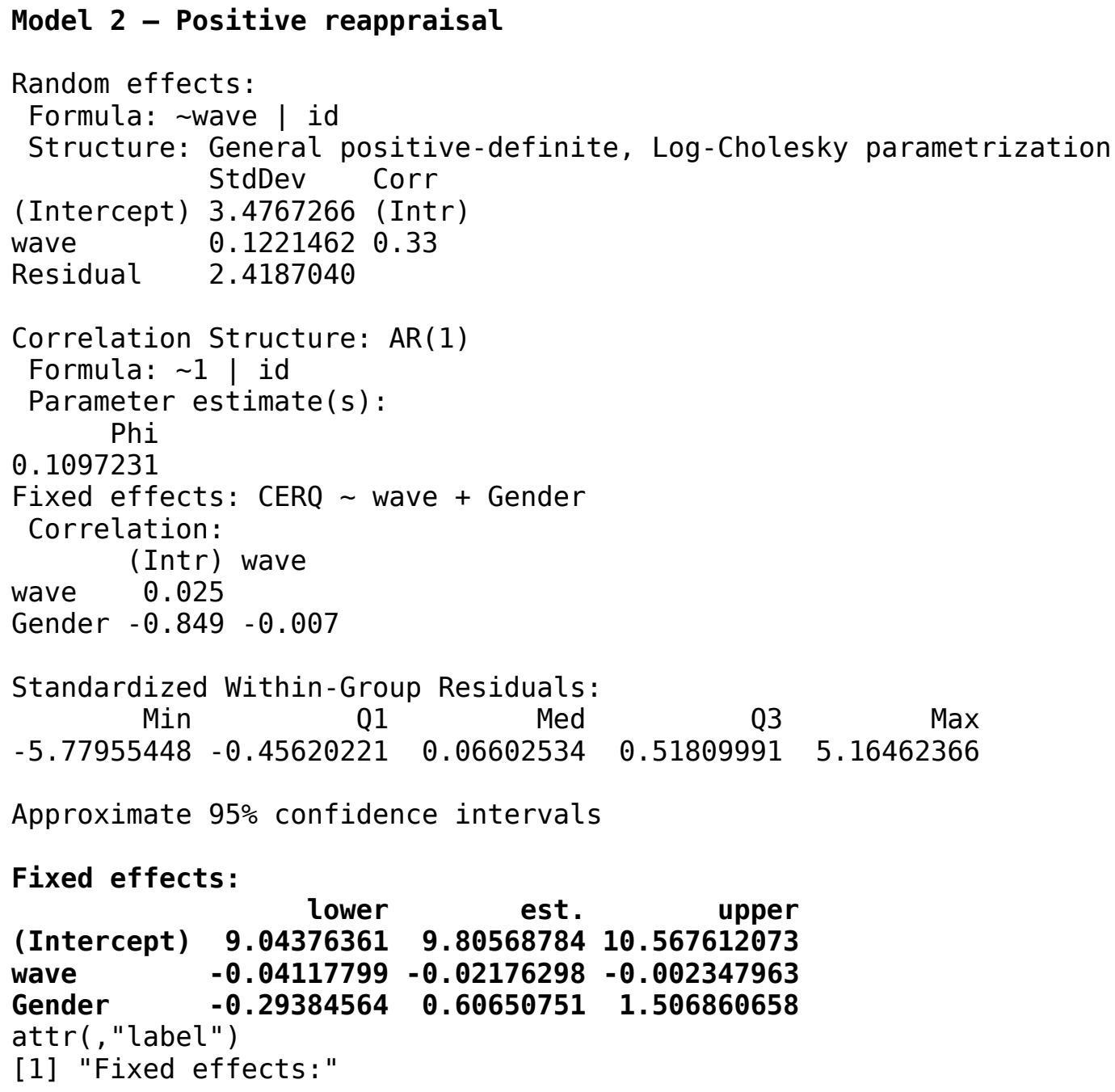




\section{Random Effects:}

Level: id

$\begin{array}{rrr}\text { lower } & \text { est. upper }\end{array}$

$\begin{array}{llll}\text { sd((Intercept)) } & 3.1882716 & 3.4767266 & 3.7912791 \\ \text { sd(wave) } & 0.1048318 & 0.1221462 & 0.1423204\end{array}$

cor((Intercept), wave) $0.1455164 \quad 0.33001340 .4923290$

Correlation structure:

lower est. upper

Phi 0.07812939 0.1097231 0.1410967

attr (, "label")

[1] "Correlation structure:"

Within-group standard error:

lower est. upper

$2.3684542 .418704 \quad 2.470020$ 